\title{
Programa de normalización de estudios previos y control de calidad en las intervenciones:
}

\author{
Metodología de estudio sobre morteros \\ en edificios históricos (2 ${ }^{\mathrm{a}}$ parte). \\ Morteros de intervención. Áridos.
}

Esther Ontiveros Ortega

Departamento de Análisis

Centro de Intervención del IAPH

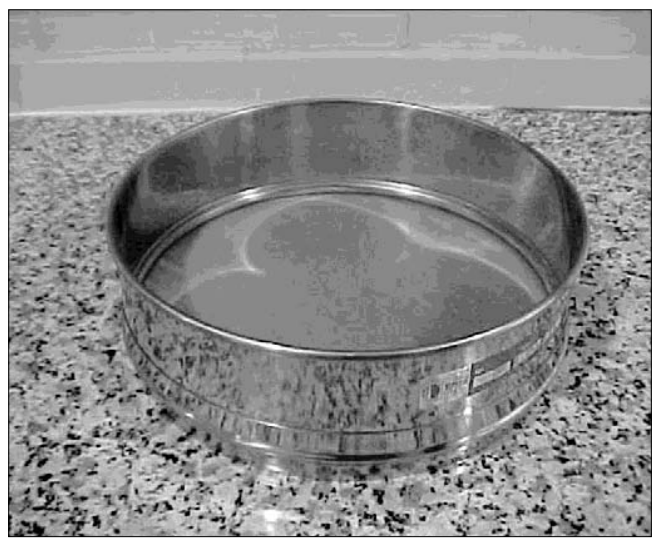

\section{Resumen}

Con este artículo se da comienzo el estudio de los morteros de intervención, tomando como base la metodología presentada en el boletín n 37 del PH. El estudio sobre estos materiales comprende el análisis de la materia prima (áridos, aglomerante y agua) y la mezcla de estos componentes en sus diferentes estados que van desde la pasta fresca, proceso de endurecimiento y estado sólido.

Las características de los componentes esenciales de este material tienen un papel decisivo en su fabricación, por tanto es importante que exista un control riguroso de estos elementos y continuas mejoras en la tecnología de su producción; de ahí la investigación en la definición, evaluación, viabilidad y comportamiento de estas materias primas.
Se va a comenzar por los áridos, apartado complejo e importante en la caracterización de los morteros. Esta metodología puede ser aplicada tanto a morteros de intervención como a morteros antiguos, en este último caso con ciertas matizaciones.

\section{Palabras claves}

Árido / Morteros de intervención / Granulometría / Coeficiente de forma / Terrones de arcilla / Equivalente de arena / Azul de metileno / Desgaste de la grava / Coeficiente de friabilidad de la arena

\section{INTRODUCCIÓN}

Se continúa con el Subprograma de Normalización de Estudios Previos y Control de Calidad en las Intervenciones: metodología de estudio sobre morteros de edificios histórico, comenzando con la caracterización de los morteros de intervención y entrando en las determinaciones a llevar a cabo sobre la materia prima (ver figura I). 
Figura 1. Metodología de estudio aplicada a morteros de Intervención

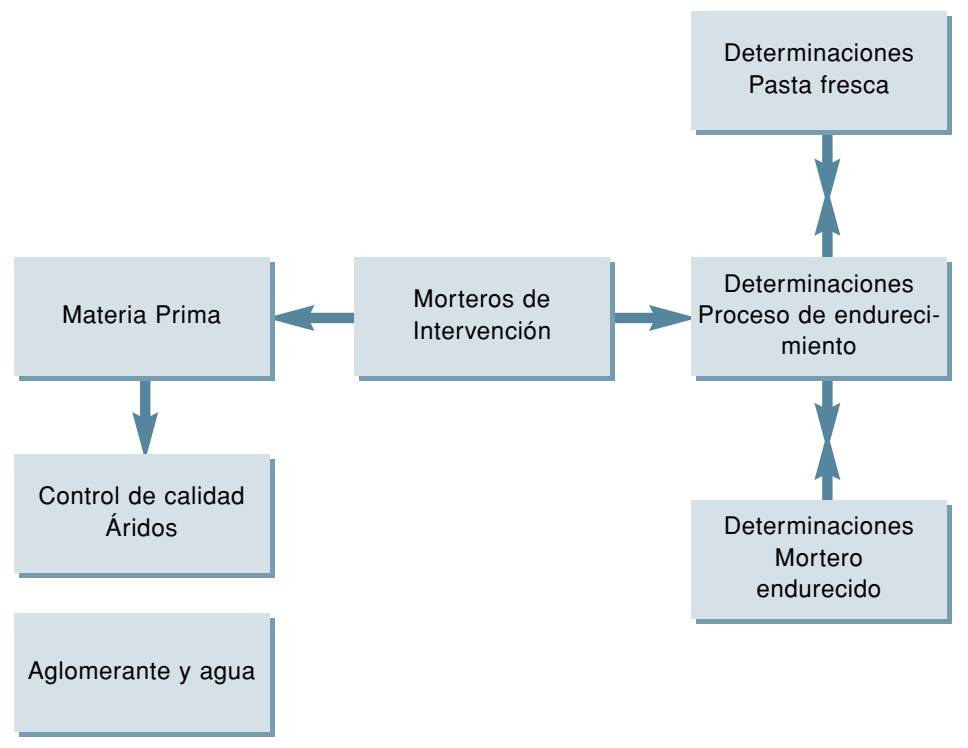

Respecto al control de calidad de estos materiales de construcción, en España se dispone de una amplia variedad de normas, lo que a veces parece ser causa de confusión entre los técnicos. Entre ellas podemos destacar las Normas Básicas de Edificación (NBE), las Instrucciones (EH-88, 91 y 99, EP-80, etc), los Pliegos ( RC-88, RY-85, etc), las normas UNE, las normas Tecnológicas de la Edificación (NTE), etc, y a esto hay que añadir las normas internacionales como ASTM, DIN, DINOS, NF, BS, ISO, Rilem, etc.

La normativa española en vigor prescribe, entre otros aspectos, los requisitos que deben cumplir los materiales empleados en la elaboración de los morteros y hormigones (de cemento), por lo tanto su aplicación a nuestro caso concreto (morteros y hormigones de cal) no está exenta de matizaciones.

Se van a estudiar los distintos ensayos empleados para la caracterización de estas materias primas en base a la normativa existente, teniendo en cuenta las condiciones óptimas especificadas por ella; comenzando por los áridos.

Como ya se indicó en el artículo del Boletín nº 34 del $\mathrm{PH}$, los áridos se definen como materiales fragmentarios "inertes" que se utilizan para la fabricación de morteros y hormigones. Al ocupar aproximadamente las 3/4 partes del mortero u hormigón, ejercen una influencia decisiva en la resistencia y durabilidad de este material, determinando en gran medida el consumo del aglomerante y el coste del producto resultante.

Estos materiales se pueden clasificar en función de las técnicas utilizadas en su aprovechamiento: áridos naturales o granulares (se trata de material detrítico o rocas detríticas poco consolidadas con modificación en la distribución de su tamaño) y árido de machaqueo (obtenido mediante la trituración, molienda y clasificación por tamaños de diferentes rocas de cantera).
También se puede hablar de: áridos ligeros (productos naturales o artificiales empleados para la obtención de elementos en obras ligeras), áridos artificiales (subproductos de procesos industriales como escorias o material de derribos), gangas de las explotaciones mineras (obtenidas de las labores de preparación en la minería subterránea y como fuente importante de materiales para áridos) y por último cenizas volantes (utilizadas como áridos o aditivos).

Las propiedades de los áridos derivan de su concepción como elemento aislado o como conjunto. Como "elemento aislado" se incluye propiedades macroscópicas físicas (dimensiones, forma, redondez, densidad, propiedades superficiales, porosidad, permeabilidad, dureza superficial, conductividad térmica y dilatación) y químicas (solubilidad, alterabilidad e hinchamiento).

Estas propiedades se ven afectadas, para el caso de áridos naturales, de las características de sedimentación y para áridos artificiales de las técnicas de machaqueo. Además influyen la composición mineralógica, la distribución, hábito, morfología de estas especies mineralógicas, grado de ordenamiento del retículo cristalino, porosidad primaria, recristalización, porosidad secundaria y accesibilidad de la porosidad.

Estos aspectos son tan importantes en los áridos que existen clasificaciones basadas en estos criterios, dentro de las que podemos destacar la clasificación de Figg y Bowden (1970) que aunque fundamentada en criterios petrográficos es a grandes rasgos química (en función de su reacción frente a los ácidos).

Dentro del "conjunto de los áridos" podemos destacar los aspectos composicionales (monogénicos y poligénicos) y propiedades morfológicas como la esfericidad, redondez y distribución del tamaño.

La esfericidad de los granos es un carácter vinculado a los factores petrogénicos como son la esquistosidad, pizarrosidad, estratificación, etc. Estos aspectos dependen de las propiedades petrofísicas de la roca original y en su caso del ambiente sedimentogenético.

Se define redondez, como la medida del filo o angulosidad relativas a los bordes o esquinas de una partícula, está directamente relacionada con la mecánica de fluidos del medio de sedimentación, la intensidad del transporte, la distribución del tamaño, la dureza del material, etc. Es una propiedad que influye en el rozamiento entre granos y afecta a la trabajabilidad y rugosidad de los morteros y hormigones. Su incidencia en los áridos es tan importante que las normas británicas, BS, (parte Ia ${ }^{\text {, } 1975) ~ p r o p o n e n ~ u n a ~ c l a s i f i-~}$ cación basada en la forma de las partículas.

Las clasificaciones de áridos más utilizadas actualmente se basan en criterios de tamaño, incidiendo de forma importante en la calidad del mortero y hormigón. La "ley de Feret" dice que cualquiera que sea la naturaleza, tamaño de los áridos, proporciones, consistencia y grado de cohesión, la resistencia de los morteros depende de la siguiente relación: 


$$
R=k\left[\frac{C}{a+c+h}\right]^{2}
$$

donde:

R: resistencia de los morteros y hormigones,

a: volúmenes ocupados por el agua,

c: volúmenes ocupados por el aglomerante,

h: volúmenes ocupados por los huecos.

K: Cte C: Cte.

El mortero será más resistente si el volumen de huecos y contenido en agua es mínimo, el porcentaje de huecos se puede reducir aumentando la distribución en el tamaño del árido. Por esta razón los áridos en conjunto deben poseer una buena adherencia con la pasta del aglomerante que permitan un mínimo de huecos en su normal apilamiento, manteniendo la homogeneidad del hormigón fresco durante la manipulación y una hidratación del aglomerante en pasta o endurecido.

La Instrucción EH establece una clasificación de áridos en función del tamaño: distingue entre "árido fino", aquel que pasa por el tamiz 5 UNE 7050, "árido grueso" el retenido por dicho tamiz, "árido total" aquél que por sí o por mezcla posea la proporción adecuada para elaborar hormigones o morteros y por último define los finos o polvo como las partículas inferiores a 0,08 $\mathrm{mm}$. Esta Instrucción tiene en cuenta parámetros como el "tamaño máximo de un árido" que lo define como la mínima abertura del tamiz UNE 7050 por el que pasa el $90 \%$ en peso y el total por el tamiz de abertura doble y "tamaño mínimo de un árido", como la máxima abertura del tamiz UNE 7050 por el que pasa menos del 10\% en peso.

\section{CONTROL DE CALIDAD SOBRE ÁRIDOS}

Las primeras normas españolas están relacionadas con la normativa aplicada en construcción y datan del 1944 (Orden Ministerial del 20 de Marzo). No será hasta 1968 cuando se apruebe la Instrucción y se constituya la Comisión Permanente del Hormigón, con el objeto de estudiar los avances científicos y tecnológicos en este campo, que deberá ser revisado cada 5 años. A partir de esta fecha la Instrucción se va actualizando con relativa periodicidad y en su elaboración se recoge la siguiente normativa UNE (ver tabla I).

\section{Tabla I. Normativa UNE aplicada a áridos}

NORMATIVA UNE

CONTENIDO

\begin{tabular}{|c|c|}
\hline $7.082-54$ & Determinación aproximada de materia orgánica en arenas para hormigones y morteros. \\
\hline 7.083-54 & Determinación del peso específico y absorción de gravas y arenas. \\
\hline 7.133-58 & Determinación de los terrones de arcilla en áridos para la fabricación de morteros y hormigones. \\
\hline 7.134-58 & Determinación de partículas blandas en áridos gruesos para hormigones. \\
\hline $7.135-58$ & Determinación de finos en áridos utilizados para la fabricación de hormigones. \\
\hline $7.136-58$ & Estabilidad de áridos frente a disoluciones de sulfato sódico o magnésico. \\
\hline 7.139-58 & Análisis granulométrico de áridos \\
\hline $7.140-58$ & Determinación de los pesos específicos y absorción de agua en áridos finos. \\
\hline 83.115-89 (experimental) & Áridos para hormigones. Medida del coeficiente de friabilidad de las arenas. \\
\hline $83.116-90$ & Áridos para hormigones. Determinación del coeficiente de los Ángeles. \\
\hline $83.120-88$ & Áridos para hormigones. Determinación cuantitativa de los compuestos de azufre. \\
\hline $7.238-71$ & Determinación del coeficiente de forma de los áridos gruesos empleados en la fabricación de hormigones. \\
\hline $7.244-71$ & Determinación de partículas de bajo peso específico que puede contener el árido utilizado en hormigones. \\
\hline $7.245-71$ & Determinación de los compuestos de azufre contenidos en los áridos. \\
\hline 7.295-76 1R. & $\begin{array}{l}\text { Determinación del contenido, tamaño máximo característico y módulo granulométrico del árido grueso en el } \\
\text { hormigón fresco. }\end{array}$ \\
\hline $83.100-84$ & Áridos para hormigón. Clasificación en fracciones granulométricas para su empleo \\
\hline $\begin{array}{l}83.101-90 \\
\text { normales.83.101-90 }\end{array}$ & $\begin{array}{l}\text { Áridos para hormigones. Clasificación en fracciones granulométricas para su empleo en hormigones } \\
\text { Áridos para hormigones. Clasificación en fracciones granulométricas para su empleo en hormigones normales. }\end{array}$ \\
\hline $83.108-90$ & Áridos para hormigones. Elementos para la identificación. \\
\hline 83.109-85 & Áridos para hormigones. Toma de muestras. \\
\hline $83.111-87$ & $\begin{array}{l}\text { Áridos para hormigones. Determinación de la resistencia a compresión de rocas empleadas en la fabricación } \\
\text { de áridos. }\end{array}$ \\
\hline
\end{tabular}




\begin{tabular}{ll}
\hline NORMATIVA UNE & \multicolumn{1}{c}{ CONTENIDO } \\
\hline 83.121-90 & Áridos para hormigones. Determinación de la reactividad árido-álcalis (método químico \\
\hline 83.124-90 (Experimental). & Áridos para hormigones. Determinación cuantitativa de cloruros. Método volumétrico. \\
\hline $83.130-90$ & Áridos para hormigones. Ensayo de azul de metileno. \\
\hline $83.131-90$ & Áridos para hormigones. Determinación del equivalente de arena. \\
\hline $83.133-90$ & $\begin{array}{l}\text { Áridos para hormigones. Determinación de las densidades, coeficiente de forma y contenido de agua en el } \\
\text { árido fino. }\end{array}$ \\
\hline 83.134-90 & $\begin{array}{l}\text { Áridos para hormigones. Determinación de las densidades, porosidad, coeficiente de absorción y contenido } \\
\text { en agua del árido grueso. }\end{array}$ \\
\hline
\end{tabular}

La granulometría, relacionada directamente con el tamaño del árido, es una de las determinaciones más importantes a llevar a cabo sobre estos materiales, los criterios de valoración granulométrica no aparecen incluidos en esta Instrucción hasta su última edición, recurriéndose hasta este momento a otras normas como ASTM, DIN, etc, para su correcta interpretación.

La última Instrucción EH-99 presenta pocas variaciones respecto a la anterior, establece el límite entre árido fino y grueso en $4 \mathrm{~mm}$ y contempla la valoración de las granulometrías por medio de husos.

La Instrucción EH-9/ pone de manifiesto en el artículo 7, que la naturaleza de los áridos y su preparación serán tales que permitan garantizar la adecuada resistencia y durabilidad del hormigón (aplicable también a morteros), así como las restantes características que se exijan a éste en el Pliego de Prescripciones Técnicas Particulares.

Pueden emplearse: arenas, gravas de yacimientos naturales, rocas machacadas, escorias siderúrgicas apropiadas y en general cualquier material recomendado. Para demostrar la idoneidad del material, en el caso de que no esté previamente normalizado, se deben realizar ensayos de identificación como análisis mineralógico, análisis petrográfico y análisis físico-químico.

Entre sus recomendaciones se pueden destacar:

- Los áridos de escorias siderúrgicas, deben ser estables (que no presenten silicatos inestables ni compuestos ferrosos). No se aconseja el empleo de áridos con sulfuros oxidables (pirrotina, marcasita y piritas) ni áridos blandos, friables, porosos ni nódulos de yeso. No deben contener materia orgánica y ser resistentes frente a las heladas.

- Los áridos no deben ser reactivos frente al aglomerante (cemento) ni descomponerse frente a los agentes exteriores. La presencia de sílice (ópalo, dacitas, etc.) o sustancias carbonatadas magnésicas (dolomitas) pueden provocar fenómenos expansivos en ciertas condiciones higrotérmicas y en presencia de álcalis generar procesos reactivos de tipo árido-álcalis. Los áridos presentarán reactividad potencial (R) con los álcalis del cemento si para:
- $R \geq 70$, la concentración de sílice resulta $>R$.

- $R<70$, la concentración de sílice resulta $>35+0,5 R$

- Se recomienda no utilizar áridos finos cuyo equivalente de arena (EAV) sea inferior al 75\%, para obras de ambiente I y $\mid$ y $80 \%$ para obras en ambiente III; a excepción de rocas carbonatadas cuyo límite se establece en un $50 \%$, siempre que su valor de azul de metileno sea igual o inferior a 60 gramos por cada 100 gramos de finos de forma general.

- La forma de los áridos también afectan a su calidad, no son aconsejables los áridos laminares ni aciculares en una proporción excesiva ni los áridos excesivamente gruesos.

- Se considera que un árido presenta buena calidad, en cuanto a sus condiciones físico químicas y mecánicas si reúne las siguientes características: composición química-mineralógica estable, elevada resistencia a la compresión simple, baja absorción de agua y elevada densidad.

La normativa española establece una serie de ensayos para la determinación de su calidad. Estas determinaciones basadas en la Instrucción EH-9| son de tres tipos fundamentalmente: de tipo granulométrico y coeficiente de forma (dentro de este apartado se incluyen la determinación del tanto por ciento en finos), de tipo físico-químico y físico-mecánico.

\section{Toma de Muestras}

El primer aspecto a tener en cuenta es la toma de muestras; la normativa española a este respecto , UNE 83. 109/85, tiene como objetivo establecer procedimientos normalizados para poder llevar a cabo un control del producto en la misma fuente de suministro, un control de las operaciones en el lugar de utilización y ensayos para la aceptación o rechazo de los materiales. No se contempla en esta norma la investigación de yacimientos como fuente de suministro de áridos.

El procedimiento operativo de toma de muestra varía según la fuente de suministro: cantera, depósitos de zahorra, grava y arenas naturales o estaciones suministradoras de áridos. 
La inspección de la cantera incluye el examen del afloramiento rocoso en el que hay que tener en cuenta las variaciones de estructura y color que presenten, llevando a cabo un muestreo en los diferentes lugares. Por otra parte se realizarán ensayos indicativos de los diferentes materiales que puedan aparecer.

Cuando se trate de depósitos de zahorra, grava y arenas naturales se llevará a cabo un examen de los diferentes componentes que constituyen el depósito, anotando las proporciones de las muestras.

Cuando se trate de áridos procedentes de estaciones suministradoras, el procedimiento operativo consiste en separar el árido en tantas unidades como se considere necesario en función de los ensayos a llevar a cabo. Cada muestra bruta se reduce a la muestra de envío para el laboratorio según los siguientes métodos:

- mediante el uso de un aparato divisor de muestras, - mediante cuarteo manual,

- y mediante el uso de aparatos divisores de muestras fillers.

\section{Análisis Granulométrico y coeficiente de forma}

\section{Análisis Granulométrico}

La "granulometría de un árido" se define como la distribución por tamaños de las partículas que lo constituyen y representa las proporciones de los granos de cada tamaño que hay en su conjunto. Ésta depende del tamaño de sus granos más gruesos o tamaño máximo, de ahí la necesidad de estimar previamente dicho tamaño.

Esta propiedad tiene una influencia decisiva en la trabajabilidad de los morteros e, indirectamente, en su durabilidad; de ahí que se aconseje en su empleo una granulometría de amplio rango o distribución. La presencia de una granulometría continua no es indicativo de la calidad de un árido, los parámetros que controlan su particularidad son la presencia de arena de finura adecuada así como la relación arena-grava.

El análisis granulométrico del árido se puede realizar separándose en fracciones o mezclado, de acuerdo con los siguientes pasos:

- Se determina el " tamaño máximo del árido", en esta fase se incluyen la determinación del tamaño máximo de la grava y tamaño mínimo de las distintas fracciones.

- Posteriormente se realizará un análisis granulométrico de ambos áridos de acuerdo con el método de la norma UNE 7|39. Los datos obtenidos se expresarán en la tabla de valores y también se expresarán los resultados de ambas granulometrías en forma de gráficos granulométricos.
- Por último se pasará a la valoración de dichas granulometrías.

I. Determinación del tamaño máximo de un árido. Este parámetro representa el tamaño de sus granos más gruesos, se relaciona el tamaño con la abertura del tamiz más pequeño por el que pasan todos o casi todos los granos de acuerdo con una serie escalonada de tamices.

Se establece el concepto de "casi todos los granos", respecto a cuánto puede quedar retenido sobre un tamiz, para relacionar su abertura con el tamaño máximo. No existe un acuerdo absoluto entre diversos autores o normas que han tratado el tema (tabla II).

Las diferencias entre la definiciones dadas por los diferentes autores no son grandes y el que existan distintas definiciones de tamaño no debe ser motivo de confusión, si se decide seguir un procedimiento u otro se debe indicar el autor.

Interesa determinar el tamaño máximo de los áridos, porque en nuestras normas existen limitaciones del mismo para la fabricación de morteros y hormigones. Por ello es conveniente emplear la serie de tamices más completa que pueda disponerse. La serie normalizada en España, especificada en la norma UNE 7050, incluye tamices cuyas aberturas se encuentran escalonadas mediante el factor ${ }^{3} \sqrt{ } 2$, de tal modo que cada tres tamices la abertura se hace doble o mitad, si es en sentido descendente (ver tabla III) .

Tabla II. Definición de tamaños según diferentes autores y normas

\begin{tabular}{ll}
\hline Autor & \multicolumn{1}{c}{ Definición } \\
\hline Instrucción EH-91 & $\begin{array}{l}\text { Luz de malla del tamiz por el que pasa, al menos, el } 90 \% \text { del árido, } \\
\text { debiendo pasar totalmente por un tamiz de malla doble. }\end{array}$ \\
\hline Bolomey & $\begin{array}{l}\text { El más pequeño de la serie que retenga menos del } 15 \% \text { del peso total del } \\
\text { árido, excluyendo los grandes cantos de dimensiones anormales. }\end{array}$ \\
\hline Faury & $\begin{array}{l}\text { Diámetro de los orificios del tamiz (ideal) más pequeño que deje pasar } \\
\text { todo el árido. }\end{array}$ \\
\hline Fuller & Igual definición que la Bolomey. \\
\hline García Balado & $\begin{array}{l}\text { Abertura de la malla cuadrada a través de las cuales pueda pasar el } 95 \%, \\
\text { en peso, del material. }\end{array}$ \\
\hline de la Peña & $\begin{array}{l}\text { Abertura del tamiz más pequeño de la serie utilizada que retenga menos } \\
\text { del } 25 \% .\end{array}$ \\
\hline
\end{tabular}

Tabla III. Serie de tamices según la norma UNE 7.050 (en milímetros)

\begin{tabular}{lllllllllll}
\hline 50 & 40 & 32 & 25 & 20 & 16 & 12,5 & 10 & 8 & 6,3 & 5
\end{tabular}

La porción de muestra que se ensaye para el análisis granulométrico va a depender del tamaño máximo del árido y su determinación se realiza mediante los cálculos que se recogen en la norma. 
2. Análisis granulométrico. Como viene indicado en la norma UNE 7 I39, para la realización del ensayo se toman mediante cuarteado $500 \mathrm{~g}$ de muestra omitiendo el mezclado en húmedo del árido fino. Este método no es válido para áridos combinados con material bituminoso ni para las cargas minerales -fillers-.

Una vez determinado el tamaño máximo (ver tabla IV). La muestra, previo al tamizado, se seca en una estufa y se separa el árido en arena y grava, pasándose posteriormente al análisis granulométrico de las fracciones por separado, según la serie de tamices indicada en la tabla V. De esta forma se calcula la proporción de arena y grava en el árido inicial, dicha proporción servirá para calcular la granulometría del árido total.

El tamizado se efectúa con ayuda de un vibrador que mantenga un continuo movimiento sobre la superficie de los tamices hasta que pase menos de un $1 \%$ del residuo retenido. No debe emplearse procedimiento o dispositivo alguno para que las piedras y granos se orienten o giren con mayor facilidad por los orificios de los tamices.

\begin{tabular}{cc}
\hline \multicolumn{2}{l}{ Tabla IV. Cantidad de material necesario } \\
\hline $\begin{array}{cc}\text { Tamaño máximo del } \\
\text { árido (mm). }\end{array}$ & $\begin{array}{c}\text { Peso mínimo de la } \\
\text { muestra (gramos). }\end{array}$ \\
\hline 10,2 & 1000 \\
\hline 12,5 & 2500 \\
\hline 20,0 & 5000 \\
\hline 25,0 & 10000 \\
\hline 40,0 & 15000 \\
\hline 50,0 & 20000 \\
\hline 63,0 & 26000 \\
\hline 80,0 & 30000 \\
\hline 100,0 & 35000 \\
\hline
\end{tabular}

Tabla V . Serie de tamices UNE 7.050

\begin{tabular}{cc}
\hline Grava & Arena \\
\hline 80 & 2,5 \\
\hline 40 & 1,25 \\
\hline 20 & 0,63 \\
\hline 10 & 0,32 \\
\hline 5 & 0,16 \\
\hline
\end{tabular}

Los resultados del análisis granulométrico se expresan como:

a. Porcentajes totales del material retenido por cada tamiz y cedazo (apreciación 0.1\%).

b. Porcentajes totales que pasan por el tamiz o cedazo.
La granulometría del conjunto se expresa como "porcentaje que pasa" y se calcula a partir de los porcentajes que pasan por las distintas fracciones, de acuerdo con lo que se indica a continuación.

I. Si el árido se ha suministrado mezclado, la granulometría total se calculará a partir de los datos de la granulometría de la arena y de la grava así como de la porción de arena y grava en el conjunto, según la siguiente resolución:

$$
T_{\mathrm{ti}}(\%)=T_{\mathrm{ai}} \cdot A \%+T_{\text {gi }} \cdot G \%
$$

donde:

$\mathrm{T}_{\mathrm{ti}}(\%)$ representa el tanto por ciento que pasa en cada tamiz para el conjunto de la muestra,

$\mathrm{T}_{\mathrm{ai}}$ es el tanto por ciento que pasa por cada tamiz para la arena,

$\mathrm{A}(\%)$ es el tanto por ciento de arena en el conjunto de la muestra,

$\mathrm{T}_{\text {gi }}$ es el tanto por ciento que pasa por cada tamiz para la grava y

$G(\%)$ es el tanto por ciento de grava en el conjunto de la muestra.

2. Si el árido ya está en fracciones independientes, se hace la mezcla en las proporciones adecuadas y se calculan los datos designados como "mezcla propuesta" de forma análoga a la indicada para el " árido conjunto".

3. Con los datos de los "porcentajes retenidos acumulados" se determina un nuevo parámetro designado como "módulo granulométrico". Su valor define la finura del conjunto de granos y representa la centésima parte de la suma de estos porcentajes "retenidos acumulados" sobre cada tamiz (a excepción del fondo). De esta forma se calcula el módulo granulométrico de la fracción arena, grava, árido total y mezcla de fracciones.

Estos valores se representan gráficamente, tanto la granulometría de los áridos por fracciones como del conjunto, en forma de "tanto por ciento en peso que pasa" (en ordenadas), a escala lineal, frente a la "abertura de tamices" a escala logarítmica (en abscisas).

Este método concuerda con la ASTM C 136-46 y con la E-2.22 c del ITCC.

3. Valoración de las granulometrías. El objetivo esencial del análisis granulométrico es dictaminar si un árido presenta una buena gradación por tamaños con el fin de poder elaborar un buen mortero u hormigón (Valverde Espinosa, 1993), de ahí lo importante que es seleccionar unos criterios adecuados de valoración.

Los criterios de valoración que se utilizan son de tres tipos fundamentalmente:

3.I Las granulometrías pueden calibrarse comparándola con la "Granulometría ideal" ejemplo Fuller, Faury, Abrans, Bolomey, etc. 
3.2 Valoración mediante el "módulo granulométrico". En este sentido podemos destacar el criterio basado en la teoría de "Abrams del módulo granulométrico" (MG), según la cual una granulometría es apta cuando el área comprendida entre la línea granulométrica de referencia y la del árido en cuestión, situada por encima de la misma, sea sensiblemente igual a la situada por debajo. Este método tiene el inconveniente de que no puede cuantificarse la mayor o menor aproximación de la granulometría del árido a la considerada como ideal.

Abrams proponía una granulometría ideal (en función del tamaño del árido) teniendo en cuenta, además, la cantidad de aglomerante. Este autor fue el primero en señalar que los áridos con igual módulo granulométrico proporcionan morteros y hormigones de igual resistencia.

Las normas francesas (NF) suelen tomar como punto de referencia para calibrar la granulometría de los áridos, sobre todo las arenas, el "módulo granulométrico"; este parámetro depende del tamaño de las partículas y da una idea de cómo están distribuidas las distintas proporciones que componen un árido o fracción, siempre que su tamaño máximo sea conocido.

El método tiene la ventaja de poder expresar el conjunto de las fracciones mediante un solo valor, el cual puede definir y valorar una determinada granulometría. Un MG de valor bajo indica una alta proporción de fracciones finas y un valor alto una alta proporción de fracciones gruesas.

Según Dreux (1981) las arenas atendiendo al MG se pueden agrupar en tres grupos (ver figura 2).

Como se refleja en tabla VI, dentro del grupo A se engloban arenas de utilización preferente porque permiten obtener a un mismo tiempo una buena docilidad y resistencia, sin riesgo de segregación. El grupo B señala arenas de menor resistencia pero buenas para la colocación en obra y por último el grupo C nos muestra arenas con altas resistencias aunque con riesgo de segregación y menor maleabilidad.

La valoración de una granulometría atendiendo al "módulo granulométrico" es aconsejable para un árido separado en fracciones, siempre que una de las fracciones sea exactamente arena; si la fracción arena presenta un buen módulo granulométrico, la aptitud de la granulometría del conjunto está asegurada porque podrá mezclarse con la grava en el porcentaje que se desee.

3.3 A través de husos granulométricos. Teniendo en cuenta la dificulta que entraña hacer una valoración sobre una línea granulométrica o sobre un solo valor, como el módulo granulométrico, se hacía necesario delimitar un intervalo en el que debieran situarse las granulometrías aptas para la fabricación de morteros y hormigones. La norma alemana DIN I045, aceptada por todos los países Europeos, incluidas las nor-
Figura 2. Husos definidos por las granulometrías

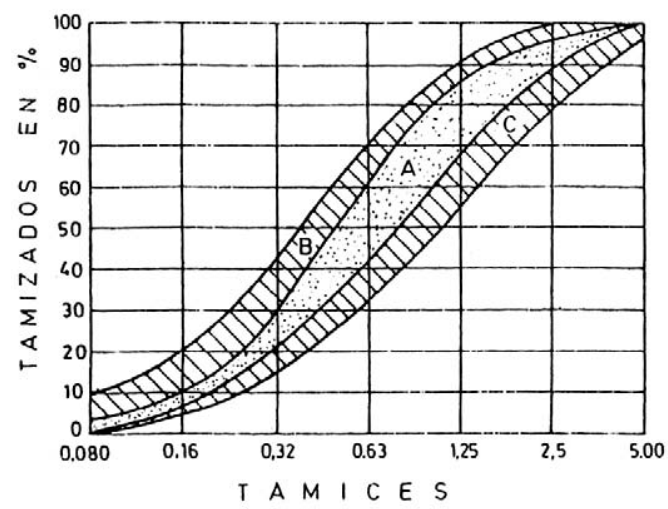

Tabla VI. Clasificación de las arenas según Dreux

\begin{tabular}{ccc}
\hline Arenas admisibles & Husos & Módulos granulométricos \\
\hline Preferente & A & 2,20 a 2,80 \\
\hline Demasiado fina & B & 1,80 a 2,20 \\
\hline Demasiado gruesa & C & 2,80 a 3,20 \\
\hline
\end{tabular}

mas UNE han definido lo que se conoce como "husos granulométricos". Para su empleo puede tomarse como tamaño máximo el definido según la Instrucción EH-91. Los husos seguidos adaptados a la serie de tamices vienen indicados en la figura 3.

Para granulometrías continuas, los husos serán favorables cuando estas curvas estén comprendidas entre la línea $A$ y $B$ y husos útiles cuando estén comprendidos entre B y C; para las granulometrías discontinuas, las curvas favorables deben estar comprendidas entre las líneas $U$ y $C$.

\section{Coeficiente de forma}

Se define este parámetro como la relación entre el volumen real del grano y el volumen de una esfera que puede circunscribirse sobre él, mide la diferencia entre la forma real de los granos del árido y la forma esférica. Esta propiedad no depende del proceso de machaqueo ni de su carácter rodado. La descripción del ensayo se contempla en la norma UNE 7238 y ésta indica que el "coeficiente de forma del árido grueso" no debe ser inferior a 0,15.

Las muestras para la realización del ensayo, deben contener un mínimo de 20 granos de cada fracción, obtenidos por cuarteo de una muestra representativa de mayor volumen. Para los áridos con mayor dispersión, se tomará mayor cantidad de muestra.

Debe determinarse el volumen de cada grano y su dimensión máxima. Según la norma el coeficiente de forma de un árido, a partir de un conjunto de $\mathrm{n}$ granos, se calcula por la siguiente expresión: 


$$
x=\frac{v_{1}+v_{2}+\ldots . . .+v_{n}}{\frac{\pi}{6}\left(d_{1}^{3}+d_{2}^{3}+\ldots . .+d_{n}^{3}\right)}
$$

donde:

$\boldsymbol{x}$ es el coeficiente de forma

$v_{i}$ es el volumen de cada grano

$\mathrm{d}_{\mathrm{i}}$ es la mayor dimensión de cada grano, es decir, las distancias entre los dos planos paralelos y tangentes a este grano que estén más alejados entre si, de entre todos los que sea posible trazar.

Esta norma concuerda con la norma AFNOR P-|8-30|.

\section{Determinación de finos en áridos}

Se entiende por finos, según la definición dada por la EH-9I, la fracción de árido que pasa a través del tamiz 0,080 UNE 7050 de tamaño inferior a $80 \mu \mathrm{m}$. Su definición no discrimina la naturaleza del mismo pudiendo aparecer como partículas sueltas o adheridas a los granos. Distintas normas consideran diferentes tamaños para esta fracción.

La norma ASTM C 33 limita su presencia al 3\% de forma general siempre que su composición no sea de carácter arcilloso, para las gravas su límite debe estar en el $1 \%$ con carácter general. Las normas francesas (NF) no hacen distinción sobre la naturaleza del material y la norma DIN 4226 considera los finos como sustancias arcillosas o polvo de piedra.
La importancia de los componentes finos en los morteros y hormigones se debe a que su presencia pueden aportar aspectos tanto beneficiosos como perjudiciales a este material:

- Aspectos beneficiosos: Sirve para ocupar los huecos dejados por los granos de mayor tamaño. Son imprescindibles para lograr hormigones impermeables y su misión fundamental es asegurar la cohesión de la masa del hormigón fresco, impidiendo su fácil segregación. Debido al fenómeno epistáxico pueden constituir núcleos a partir de los cuales se orientan las redes cristalinas de los compuestos hidratados del cemento. Los finos retienen agua para su necesario mojado superficial en el amasado del hormigón, lo que puede incrementar la relación agua/cemento.

- Aspectos perjudiciales: Los finos arcillosos o micáceos se interponen entre los productos de hidratación del cemento, debilitando las uniones entre componentes del hormigón endurecido. Los finos de naturaleza semejante a la de los áridos tienen la propiedad de constituir pequeños áridos susceptibles de ser ligados por la pasta del cementante, pero las arcillas carecen de esta propiedad por no tener estabilidad de volumen y por su tamaño casi coloidal.

La cantidad de finos necesarios para evitar estos problemas será mayor a medida que aumente el módulo granulométrico de la arena y angulosidad y disminuirá cuanto mayor sea el contenido en agua y tamaño del árido.

\section{Figura 3. Husos definidos por la norma DIN}
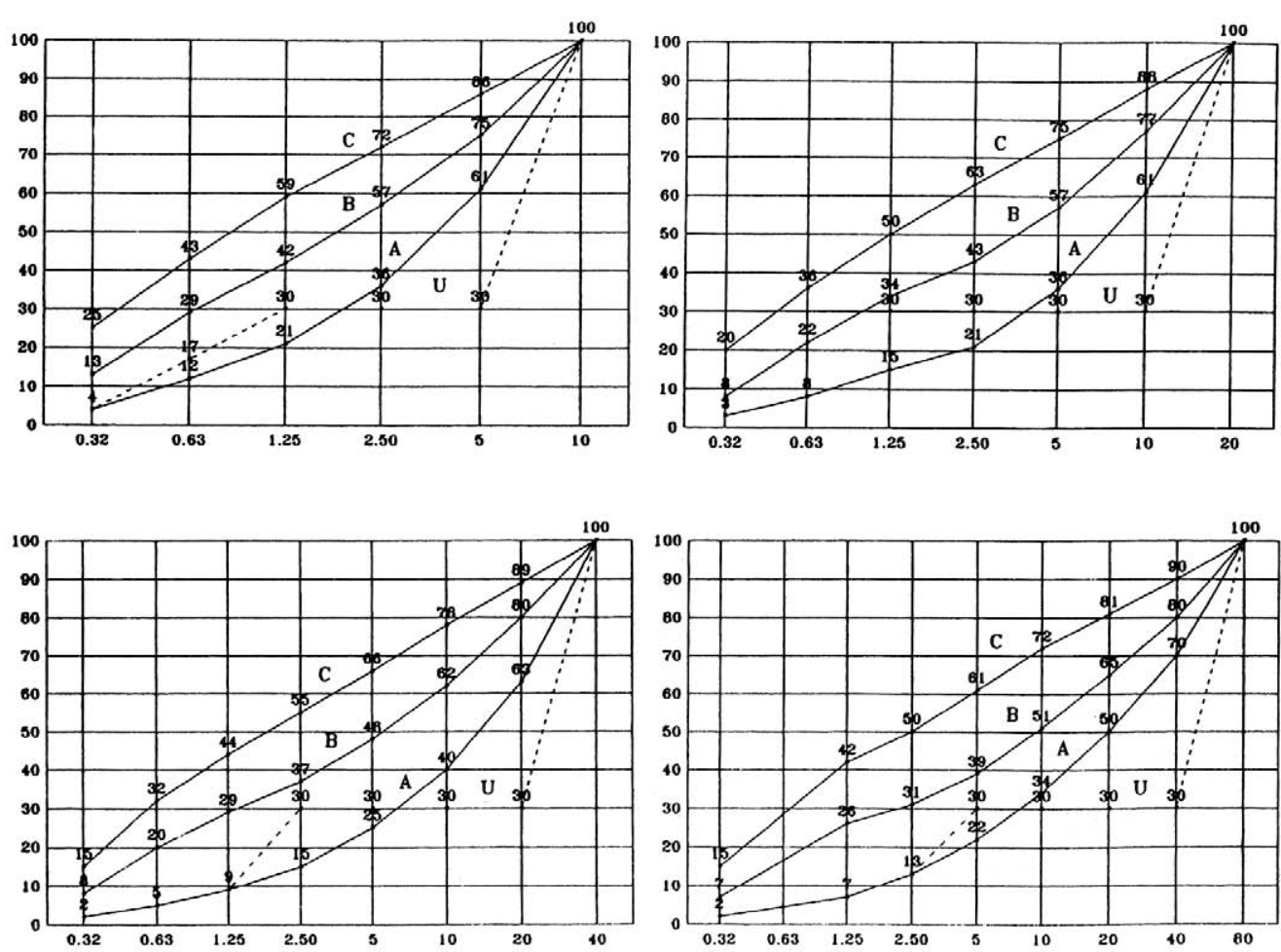
Para la determinación de los finos se ha seguido la norma UNE 7I35. El procedimiento se basa en separar mediante lavados y tamizados sucesivos las partículas finas existentes en los áridos.

Para el ensayo se toma $2,5 \mathrm{Kg}$ de muestra seca que se vierte en un recipiente al que se le añade agua hasta cubrirla totalmente, se agita la muestra hasta poner en suspensión las partículas finas. El agua junto con las partículas en suspensión se pasan a través del tamiz 0,080 UNE 7050. El proceso de lavado se repite tantas veces como sea necesario. Posteriormente la muestra ya lavada totalmente se seca en una estufa hasta peso constante.

El porcentaje de material fino que contiene el árido se obtiene con arreglo a la formula siguiente:

$$
\text { Finos } \%=\frac{P_{1}-P_{2}}{P_{1}} \times 100
$$

Donde :

$P_{1}=$ peso de la muestra original seca.

$\mathrm{P}_{2}=$ peso del material lavado seco.

Esta norma concuerda con la ASTM C I 17-49 y E I.32-c del Instituto Técnico de la Construcción y del Cemento.

\section{Condiciones físico-químicas de los Áridos}

Bajo el epígrafe "condiciones físico-químicas" se recogen aquellos compuestos asociados a los áridos, cuyo contenido queda limitado con el fin de prevenir ciertas patologías que pueden aparecer en los morteros. Estas sustancias según la Instrucción EH-9| básicamente son: terrones de arcilla, partículas blandas, partículas de bajo peso especííco, compuestos de azufre, materia orgánica, compuestos de cloro, partículas de tamaño inferior a $100 \mu \mathrm{m}$, la naturaleza de estos finos y la presencia de sustancias que puedan presentar una reactividad potencial con los áridos.

\section{Terrones de arcilla en áridos gruesos para hormigones}

Los terrones de arcilla son aglomeraciones de partículas finas, en general material arcilloso, con cierta cohesión que pueden aparecer con frecuencia asociados a los áridos. El lavado al que normalmente se somete este material, previo a su utilización, resulta insuficiente para su total eliminación.

Los efectos negativos que aporta a la pasta de los morteros y hormigones se pueden concretar en cuatro puntos fundamentalmente: gran avidez por el agua, gran capacidad de absorción, tendencia a aumentar de volumen y poca adherencia con el resto de la pasta del mortero.
La norma ASTM C-33/90 contempla bajo un mismo concepto los "terrones de arcilla" y "las partículas friables" fácilmente desmoronables atribuyéndole a ambas efectos negativos.

La norma aplicable es la UNE 7|33, que coincide con la norma con la NE 1.32-a y esencialmente con la ASTM C 142-39.

\section{Determinación de partículas blandas en áridos}

Se consideran partículas blandas aquellas que presentan una superficie de escasa dureza, fácilmente desmoronables o partículas susceptibles de ser ralladas. Su efecto negativo sobre el hormigón se centra en la defectuosa adherencia que tiene lugar entre la pasta del cementante y dichas partículas.

Dentro de este grupo se pueden incluir algunos granos de naturaleza pizarrosa o constituidos por micaesquistos blancos, la denominada greda (aglomeraciones de partículas pequeñas con poca cohesión), ciertos conglomerados naturales débilmente cementados, y por supuesto, los terrones de arcilla y partículas friables.

Las partículas blandas se limitan en la Instrucción EH9 I sólo en el árido grueso a un 5\%. No existe limitaciones específicas a este respecto en la normativa ASTM, NF y DIN.

La normativa española UNE 7 I 34 contempla la determinación de dicho parámetro con el objetivo de describir un método de ensayo aplicable tanto para la identificación de partículas blandas en toda la masa, como para poner de manifiesto la posible existencia de recubrimientos de escasa dureza sobre los áridos.

El método se basa en la resistencia al rayado que presentan las partículas del árido, para lo que se usa un esclerómetro (un cilindro de cuzín de 1,5 mm de diámetro con dureza Rockwell comprendida entre B.65 y B.67) y un conjunto de cedazos. Se considerará que un grano es blando cuando se produzca un surco por efecto del rallado.

A la hora de expresar los resultados se indican: peso y número de las piedras de cada tamaño que se han ensayado, peso y número de las piedras de cada tamaño calificadas como blandas, porcentaje de elementos blandos (en peso y número de cada tamaño), porcentaje medio ponderal de elementos blandos (referido a la muestra original, sin tener en cuenta el tamaño inferior a $10 \mathrm{~mm}$ ). Todos estos porcentajes se expresan con dos cifras decimales.

El ensayo tiene correspondencia con la norma ASTM C 235-47T y la E I32-b del Instituto Técnico de la Construcción y el Cemento. 


\section{Materia orgánica en arenas para morteros y hormigones}

Se entiende por materia orgánica, aquellas sustancias de esta naturaleza capaces de producir alteraciones en los procesos de fraguado y endurecimiento de los morteros. El material orgánico que con más frecuencia aparece en los áridos se encuentra en forma de ácido húmico y especialmente ácido fúlvico, ambos originados generalmente en las arcillas portadoras de humus.

Según Hummel (1966) la distribución de la materia orgánica influye de un modo más determinante que su concentración. Neville (1984) estudió la resistencia de los morteros en función de su contenido en materia orgánica, comprobando que en el seno del mortero la materia orgánica va degradándose con el tiempo, anulando su efecto negativo sobre la hidratación del cemento. Este material puede contribuir a la corrosión acelerada de las armaduras debido a la disminución de la basicidad de la pasta del aglomerante cuando reacciona con la cal liberada durante la hidratación de la materia orgánica. La retirada de parte de la de cal, disminuye la alcalinidad del mortero dificultando la pasividad del acero.

La normativa española UNE 7082 muestra la descripción del ensayo basado en un procedimiento colorimétrico, aplicable para su determinación aproximada.

Valverde Espinosa (1993) establece determinaciones cuantitativas de materia orgánica, utilizando la técnica propuesta por Walkley y Black y el procedimiento de ensayo establecido en la norma NLT- I 17; metodología no utilizada usualmente en áridos. Este autor pone de manifiesto que la presencia de un soluto como el hidróxido de sodio impide, incluso para muestras de un alto contenido en coloides, que se mantengan en suspensión las partículas colorantes. Esta sal confiere a la dispersión un marcado carácter floculante, eliminando el posible error del ensayo (Singer et al, 1976).

El procedimiento de ensayo establecido por la norma NLT- I 17 consiste en oxidar la materia orgánica con agua oxigenada, por diferencia entre el peso de la muestra inicial seca y el peso después del tratamiento, se calcula la porción de materia orgánica volatilizada mediante la oxidación.

Las citadas normas de ensayo proponen operar con la fracción inferior a $2 \mathrm{~mm}$ y pulverizar las muestras hasta que pasen por el tamiz 100 ASTM. En este método, aplicado a este caso concreto, incluye la fracción menor a $5 \mathrm{~mm}$. Esta norma se corresponde con la ASTM-C40-48.

\section{Determinación de partículas de bajo peso específico que puede contener el árido}

Este parámetro tiene como objetivo determinar de un modo aproximado la presencia de sustancias de baja densidad como el carbón, lignito, pequeños trozos de madera, materia vegetal, etc; que aparecen relaciona- das con sustancias de contaminación, generalmente antrópica, o como gangas de explotación minera.

Estas partículas se caracterizan por su capacidad de hincharse al absorber agua, lo cual genera unas presiones en el seno del mortero y hormigón endurecido que lo fisuran y debilitan. Muchas de estas partículas presentan los mismos inconvenientes que la materia orgánica.

En las norma ASTM C-33 este parámetro es equiparable al que se establece para el carbón y lignito, estando sus limites en 0,5\% para arenas y entre 0,5 y I,0\% para gravas; en función de los componentes del mortero y tipo de ambiente de exposición. La norma NF contempla este parámetro bajo la denominación de "Homogeneidad H" incluyendo dentro de este parámetro compuestos metálicos de bajo peso específico. La norma DIN 4266 lo considerada bajo la denominación de "materia de origen orgánico" donde además del carbón y lignito incluye el humus.

La normativa española UNE 7244 contempla este ensayo para áridos que pasen por el tamiz 5 UNE 7050. El árido grueso retenido en el tamiz 5 UNE 7050 , se homogeneiza y se tritura hasta que pase totalmente por dicho tamiz al igual que el árido fino.

Esta norma concuerda con la publicación MELC 4.02-a, del laboratorio Central de Ensayo de Materiales de Construcción de Madrid y con la norma ASTM C-123-64.

\section{Determinación de los compuestos de azufre}

Los sulfatos pueden aparecer en los áridos en forma de yeso, éste al ser un material deleznable suele estar con más frecuencia en la fracción fina, si se encuentra en concentraciones importantes termina hinchando y fisurando los morteros.

Con la aparición de la Instrucción EH-88 el contenido de sulfatos permitido se aminoró de forma considerable, en Instrucciones anteriores al 1988 el contenido en sulfatos se limitaba a 1,2\%, referido al peso seco del árido, sin embargo lo más llamativo de esta Instrucción es prohibir el empleo de áridos con sulfuros oxidables y que su determinación cuantitativa debía ser analizada siguiendo la norma UNE 83.I20.

La Instrucción EH-9| establece la norma UNE 7.24, para determinar cualitativamente la existencia en los áridos de compuestos de azufre solubles en agua o en ácido clorhídrico y sulfuros oxidables durante la hidratación. Sin embargo este procedimiento, según las últimas revisiones de la Normativa, es ineficaz para valorar el contenido en sulfuros con precisión, ya que éstos pueden volatilizarse en forma de gas sulfhídrico al tratar el ácido con ácido clorhídrico (Chinchón et al. 1989). Por esta razón Valverde Espinosa (1993) aconseja seguir la normativa UNE 83. 120 para determinar los sulfatos y sulfuros totales extraíbles 
por disgregación alcalina oxidante; esta disgregación se hace por vía seca, utilizando óxido de sodio y por vía húmeda, añadiendo una disolución acuosa saturada de bromo. La determinación posterior de sulfatos extraíbles por disgregación alcalina no oxidante, a través de un procedimiento similar al utilizado en la norma UNE 7.245, permite obtener por diferencia el contenido en sulfatos oxidables.

Cuando el ensayos cualitativo da positivo, se procede al análisis cuantitativo según la norma UNE 83.12088. Esta norma se aplica a todos los áridos empleados en la fabricación de morteros y hormigones definidos en la norma UNE 4 I- I I y UNE 4 I- I I2.

\section{Compuestos de cloro}

Se entiende por compuestos de cloro aquellas sales que pueden disociarse liberando $\mathrm{Cl}$. En lo referente a los áridos estos compuestos, debido a su carácter fuertemente soluble, pueden movilizarse y cristalizar en el seno de los morteros; pudiendo atacar a la fábrica y posibles armaduras metálicas presentes en el seno de estos materiales o proximidad.

La Instrucción EH-9I establece una limitación de estos compuestos sin establecer una especificación independiente para áridos, para su determinación se sigue la norma UNE 83.I 24 en fase experimental.

\section{Determinación del equivalente de arena, (EA)}

Esta determinación tiene como objetivo calcular la proporción y características de los finos (arcilla, polvo, etc) que contiene un suelo granular o un árido fino. El ensayo puesto a punto por Hueen (1950) para suelos fue aplicado posteriormente a áridos.

El ensayo viene recogido en la norma UNE 83.131 y NLT- I 3 y el método se basa en la ley de Stokes, porque para partículas de tamaño menor de $100 \mu \mathrm{m}$ el análisis granulométrico no resulta eficaz.

El método consiste en sedimentar una muestra de arena dentro de una probeta que contiene una disolución floculante, las partículas arenosas precipitan en el fondo mientras los finos y coloides se mantienen en suspensión. Las condiciones de ejecución son muy precisas, pues el ensayo está concebido para que pasados 20 minutos después de la agitación y posterior sedimentación de las partículas más gruesas, queden en suspensión las partículas de tamaño menor de $20 \mu \mathrm{m}$. Pese a esto se aconseja repetir la separación de las partículas varias veces, debido a que su floculación y disposición influyen de forma importante en la sedimentación.

\section{Ensayo de azul de metileno (VA)}

Este parámetro complementa la determinación anterior (EA) y tiene como objetivo conocer si los finos contienen en su composición un porcentaje impor- tante de material arcilloso. El procedimiento se basa en la capacidad de absorción del material arcilloso con respecto a ciertas sustancias (en este caso azul de metileno). Este compuesto es absorbido preferentemente por los minerales de la arcilla, la materia orgánica y los hidróxidos de hierro, siendo un indicador de su actividad superficial.

Para la realización del ensayo se sigue la norma UNE 83.130 y este método permite medir la capacidad de adsorción de azul de metileno por los finos contenidos en la arena.

Esta norma define el "valor de azul de los finos" (VA), como la cantidad de azul de metileno absorbido por cada 100 gramos de finos. Si estos finos se encuentran en baja proporción, la cantidad de muestra para la determinación debe ser alta. Esto puede causar un problema para conseguir la distribución y homogeneización de dicha disolución. Este problema se puede subsanar desechando, de las muestras del ensayo, las fracciones superiores a 2,5 mm, con un error mínimo ya que estas fracciones presentan una superficie despreciable en el conjunto de la muestra.

\section{La reactividad de los áridos con respecto a los álcalis}

Una de las propiedades de los áridos que más interés ha despertado ha sido su reactividad con los álcalis del cemento como ya se vio en el boletín $n^{\circ} 35$ $\mathrm{PH}$. Este parámetro viene contemplado en las distintas Instrucciones españolas y según se desprende de ellas y de la bibliografía existente relacionada con el tema, los áridos susceptibles de ser inestables son la silice amorfa y algunas dolomías.

La Instrucción EH-9I prescribe la evaluación de la reactividad potencial a través de la norma UNE 7I37. Se basa esencialmente en someter el material, de una granulometría preferentemente controlada, a los efectos de una disolución alcalina de hidróxido sódico a $80^{\circ} \mathrm{C}$ durante 24 horas. El método consiste en comparar la cantidad de silice que se solubiliza por este tratamiento con la reducción de alcalinidad (R) que experimenta la disolución de hidróxido sódico, tras la reacción árido-silíceo-álcalis . Cuanto más silice se solubilice y menor se reduzca la alcalinidad de la sosa $(\mathrm{NaOH})$, más sensible será el árido para producir la reacción.

Esta norma se corresponde con la norma ASTM C 283-54T y la I.T.C.C -E I.32.

Aunque este ensayo determina la sílice reactiva, la propia Instrucción advierte del riesgo que presentan otras sustancias como los carbonatos magnésicos.

En la bibliografía existen métodos que permiten cuantificar la reactividad de los áridos dolomíticos, entre ellos se puede destacar:

- Determinación del residuo insoluble. El factor más influyente en la expansión de los áridos dolomíticos es la presencia de ciertos tipos de arcillas. El método 
de ensayo viene especificado en la norma UNE 7.095 "método para la determinación del anhídrido silíceo y residuo insoluble, de los óxidos de aluminio, hierro, óxido de calcio y magnesio en cales y calizas" y consiste en tratar con clorhídrico diluido la muestra pulverizada, anotando la fracción que queda sin disolver tras la calcinación correspondiente.

- Método de la barra del mortero. El método viene especificado en la norma ASTM C.227-87 y se basa en medir la expansión lineal desarrollada en probetas fabricadas con mortero de forma prismática elaboradas con árido en cuestión y cemento con alto contenido en álcalis. Con este método se determina la posible reactividad de los áridos frente a los álcalis que normalmente tiene el cemento; basado en la reacción del árido con una disolución de hidróxido sódico en condiciones que puedan ser examinadas en laboratorio.

- Método del cilindro de roca. Este método se aplica sobre los áridos de rocas carbonatadas con la finalidad de conocer sus características expansivas. El ensayo viene especificado en la norma ASTM C.586-86 y consiste en medir los cambios de longitud que puedan presentar, a determinados plazos, pequeños cilindros de roca tras una inmersión en una disolución de hidróxido sódico a temperatura ambiente.

\section{Condiciones Físico-Mecánicas}

La Instrucción, finalmente, establece condiciones de tipo mecánico en los áridos como medida para controlar sus características de resistencia. Para medir estas condiciones incluye los siguientes parámetros: desgaste de la grava y arena, estabilidad frente a las sales, densidad, coeficiente de absorción y contenido en agua del árido.

\section{Friabilidad de la arena y desgaste de la grava}

La resistencia mecánica del árido resulta de gran importancia debido a su incidencia en la resistencia global de los morteros y hormigones. Las propiedades indicadas en este apartado muestran la resistencia de los áridos, tanto de tamaño arena como grava, a la abrasión.

En el PG-3/75 ya viene incluida la determinación al "desgaste de los Ángeles" aplicado a áridos de tamaño grava. Para morteros y hormigones estructurales, la introducción del desgaste y friabilidad en la actual Instrucción supone la intención de establecer un mínimo de calidad mecánica en los áridos.

Las normas ASTM solo contemplan este parámetro aplicado a la grava "ensayo de los Angeles", siendo en este caso más tolerante que la Instrucción EH-9|; estableciendo el límite en 50. La norma NF |8-30। fija el mismo límite y métodos de ensayo que la Instrucción EH-9I, no obstante para el árido grueso establece otro método operativo denominado "ensayo de Micro-Deval" en presencia de agua; fijando el lími- te en 35. La norma Din 4226 no contempla ninguno de estos parámetros para medir la resistencia del árido, poniendo de manifiesto que los áridos deben ser sólidos para permitir la preparación de un hormigón con las propiedades requeridas.

El ensayo especificado en la norma UNE 83 I I6 (aplicado a grava) depende directamente del grado de conexión entre las partículas, de su forma (coeficiente de forma y angulosidad), de la dureza del árido, de su mineralogía y textura (microfisuraciones, porosidad y anisotropía).

Panet y Toureq (197I) indican que este ensayo solo debe de efectuarse sobre áridos de machaqueo ya que los valores obtenidos mediante este ensayo para áridos rodados son sensiblemente inferiores.

Friabilidad de la arena (FA). El método viene recogido en la norma UNE 83.1 I 5 consiste en medir la evolución que sufre el tamaño de un árido de tamaño arena en el seno de un cilindro rotatorio, al ser sometida a una carga abrasiva en presencia de agua.

El ensayo requiere una máquina de Micro-Deval para someter a la arena a un desgaste por rozamiento. La muestra se lava (para asegurar que han quedado eliminadas las partículas menores de $0,1 \mathrm{~mm}$ ) y se introduce en un recipiente con 2,5 litros de agua y la carga abrasiva (bolas de acero). Terminado el proceso de desgaste, la evolución de la granulometría se valora por cernido en húmedo sobre el tamiz 0,050 UNE 7050. Debido a la intensa actividad superficial que presentan las partículas, se aconseja la utilización de un dispersante (hexametafosfato sódico) para facilitar el proceso.

El coeficiente de friabilidad, FA, se determina a través de la siguiente expresión:

$$
F . A=\frac{500-m}{500} \cdot 100
$$

donde:

$\mathrm{m}$ : es el peso del rechazo sobre el tamiz 0,05 mm UNE 7050 lavado y seco.

Resistencia al desgaste de la grava. Este parámetro se determina según el método que viene recogido en la norma UNE 83.1 6 y NLT | 49/72 (108). Con este ensayo se establece la resistencia a la fragmentación del árido tamaño grava como consecuencia del desgaste producido sobre una superficie metálica.

Consiste en someter a una determinada grava, previamente lavada y secada, al impacto de un determinado número de bolas de acero "carga abrasiva" en el seno de un cilindro rotatorio. El ensayo debe realizarse sobre distintas familias granulométricas cuya gama deberá ser lo más amplia posible. El coeficiente de desgaste se mide como la evolución que experimenta una granulométrica determinada sobre el tamiz I,6 UNE 7050 y su valor se obtiene de acuerdo con la siguiente expresión: 


$$
\text { Coef. de Ángeles }=\frac{M-m}{M} \cdot 100
$$

donde:

M: es el peso de la muestra ensayada

m: es el rechazo sobre el tamiz I.6 UNE 7050.

\section{Absorción de agua}

Se entiende por absorción de un árido, a la relación entre el aumento de volumen en peso que experimenta el árido (debido a la imbibición parcial de agua) y su peso seco. Esta propiedad esta directamente relacionada con la porosidad.

La porosidad tiene una enorme importancia sobre la mayoría de las propiedades de los materiales (resistencia mecánica y química, heladicidad, variaciones volumétricas con los cambios de humedad, etc). Aunque no existe una relación clara entre la resistencia del mortero u hormigón y la absorción del árido, Neville (1984) afirma que los poros de la superficie de los áridos afectan a la adherencia entre el árido y la pasta del aglomerante (cemento).

La Instrucción española en referencia a la absorción de agua contempla a su vez la determinación del peso específico cuyo proceso analítico queda reflejado en las siguientes normativas: UNE 83.133 (método del picnómetro para arenas) y UNE 83.134 (método de la balanza hidrostática para gravas).

Antes de entrar en la descripción de los ensayos hay que tener en cuenta ciertos conceptos aplicados a los áridos: peso específico real, peso específico aparente, peso específico neto de los granos y peso específico de los granos saturados con la superficie seca.

Se denomina "peso real" de una arena o grava a la relación entre el peso en el aire de un volumen dado de material, exento de poros, a una temperatura determinada y el peso en el aire de un volumen igual de agua destilada a la misma temperatura.

Se denomina "peso especifico aparente" de los áridos, a la relación entre el peso en el aire de un volumen dado de material, con inclusión de poros, a una temperatura determinada, y el peso en el aire de un volumen igual de agua destilada a la misma temperatura.

Se denomina "peso específico de los granos saturados con la superficie seca", a la relación entre el peso en el aire de un volumen dado de un árido cuyos poros estén saturados de agua y la superficie de sus granos secos, a una temperatura dada y el peso en el aire de un volumen igual de agua destilada a la misma temperatura.

Se denomina "peso específico neto de los granos" al peso específico de los granos desecados incluyendo en el volumen solo los huecos inaccesibles.

Se entiende por "coeficiente de absorción de agua de una arena o grava" a la relación entre el peso de agua que pueda absorber el árido y el peso de éste en seco, obteniéndose ambos valores en determinadas condiciones.

Método del picnómetro. El método de ensayo viene descrito en la norma UNE 83.I 33 y tiene por objetivo establecer la determinación del peso especíico y la absorción de agua en áridos de tamaño arena que cumplan las prescripciones establecidas, en cuanto al máximo contenido en finos menores de $0,080 \mathrm{~mm}$ y EV.

Esta norma concuerda con la C I28-79 de la ASTM., esta norma sustituye a la norma UNE 7.I40 así como a la parte correspondiente a la arena de la norma UNE 7.083.

Se ha experimentado otro proceso alternativo que consiste en secar la muestra dentro del propio picnómetro; para ello se hace necesario acoplar dicho aparato a una cámara de expansión. La ventaja del método consiste en que no se pierde muestra en la operación del vaciado del picnómetro y resulta muy adecuado al reducir el tiempo de ensayo considerablemente.

Método de la balanza hidrostática. El método viene recogido en la norma española UNE 83.I 34 y tiene como objetivo determinar del peso específico y la absorción de agua en áridos de tamaño grava.

\section{Estabilidad de áridos frente a disoluciones de sulfato sódico o sulfato magnésico}

La Instrucción española establece como método de ensayo la norma UNE 7I 36 para determinar la resistencia a la desintegración de los áridos al ser sometidos a ciclos alternativos de inmersión en disoluciones saturadas de sulfato sódico o sulfato magnésico y de secado a $105^{\circ}-110^{\circ} \mathrm{C}$.

El ensayo consiste en establecer una comparación entre el comportamiento de los áridos sometidos a una inmersión en soluciones saturadas respecto a su comportamiento a la intemperie. Esta comparación es muy valiosa y difiere si la solución es de sulfato magnésico o sódico.

Se precisa de un conjunto de cedazos y tamices: serie UNE 7050: 0.16, 0.32, 0.63, 1.25, 2.5, 5 - 10, $12.5,20,25,32,40,50$ y 63 , un recipiente perforado que permita el libre acceso y drenaje de la disolución sin perdida del árido ni de las disoluciones saturadas de sulfato sódico y magnésico.

Tanto los áridos pequeños como grandes deben lavarse y secarse en estufa adecuadamente antes de someterse al ensayo. Dependiendo del tamaño se procederá de la siguiente manera:

- Para el árido fino. Se pasan $100 \mathrm{~g}$ de muestra de árido total por el cedazo 10 UNE 7050, y posteriormente se tamiza por los siguientes tamices UNE 7050: de $0,16 \mathrm{~mm}$ a $0,32 \mathrm{~mm}$, de $0,32 \mathrm{~mm}$ a $0,63 \mathrm{~mm}$, de 0,63 $\mathrm{mm}$ a $1,25 \mathrm{~mm}$, de 1,25 a $2,5 \mathrm{~mm}$ y de 2,5 a $5 \mathrm{~mm}$. 
\begin{tabular}{l}
$\longleftarrow$ \\
$\square$ \\
\hline
\end{tabular}

\section{Tabla VII. Fracciones de árido grueso empleadas en el ensayo}

\begin{tabular}{lc}
\hline \multicolumn{1}{c}{ Tamices y Cedazos (UNE 7050) } & Cantidades \\
\hline $5 \mathrm{~mm}$ a $10 \mathrm{~mm}$ & $300 \mathrm{~g}$ \\
\hline $10 \mathrm{~mm}$ a $20 \mathrm{~mm}$ & $1.000 \mathrm{~g}$ \\
\hline Pasen $12,5 \mathrm{~mm}$ retenido $10 \mathrm{~mm}$ & $33 \%$ \\
\hline Pasen $20 \mathrm{~mm}$ retenido $12,5 \mathrm{~mm}$ & $67 \%$ \\
\hline $20 \mathrm{~mm}$ a $32 \mathrm{~mm}$ & $1.500 \mathrm{~g}$ \\
\hline Pasen $25 \mathrm{~mm}$ retenido $10 \mathrm{~mm}$ & $33 \%$ \\
\hline Pasen $40 \mathrm{~mm}$ retenido $25 \mathrm{~mm}$ & $67 \%$ \\
\hline $40 \mathrm{~mm}$ a $63 \mathrm{~mm}$ & $3.000 \mathrm{~g}$ \\
\hline Pasen $50 \mathrm{~mm}$ retenido $40 \mathrm{~mm}$ & $50 \%$ \\
\hline Pasen $63 \mathrm{~mm}$ retenido $50 \mathrm{~mm}$ & $50 \%$ \\
\hline
\end{tabular}

- Para el árido grueso: Se elimina la fracción que pasa por el tamiz 5 UNE 7050 y se ensayan las fracciones que se indican en la tabla VII que representen el 5\% o más del total de la muestra.

Los áridos se someten a ciclos de inmersión en la disolución durante 16 o 19 horas y secado a $\mathrm{T}^{\circ} 105^{\circ}$ $110^{\circ} \mathrm{C}$ y pesado tras el previo enfriamiento. Terminado el número de ciclos y enfriadas las muestras se lavan hasta eliminar el sulfato (se añade para ello cloruro de bario al 5\%), se secan, se tamizan por el mismo tamiz y se pesan.

En este método de ensayo se indica: la granulometría de la muestra original, la cantidad de muestra que se ensaya para cada fracción, porcentaje de muestra que retiene cada fracción después del ensayo, porcentaje de muestra de cada fracción referida a la granulometría original del material, tipo de disolución que se ha empleado en el ensayo y número de ciclos a que la muestra ha sido sometida.

Esta muestra concuerda con la norma ASTM C 289$52 T$ y con la E 1.32 del Instituto Técnico de la Construcción y del Cemento.

Bibliografía

AENOR. UNE 7238. Determinación del coeficiente de forma de áridos gruesos empleados en la fabricación de hormigones. 1971.

AENOR. UNE. 7139. Análisis granulométrico de áridos. 197I.

AENOR UNE-7050. Tamices de ensayo. Definiciones de los términos utilizados en tamices y análisis granulométrico por tamizado.

AENOR. UNE. 7I35. Determinación de finos en áridos utilizados para la fabricación de hormigones. 1995.

AENOR UNE 7.I33-58. Determinación de terrones de arcilla en áridos gruesos para hormigones.

AENOR UNE 7.133-58. Determinación de partículas blandas en áridos.

AENOR UNE 7.244-7I. Determinación de partículas de bajo peso específico que puede contener el árido

AENOR UNE 7.245-7I y UNE 83. 120-88. Determinación de los compuestos de azufre

AENOR UNE 7.080-54. Determinación de la materia orgánica en arenas para morteros y hormigones.

AENOR UNE 83.131-90. Determinación del equivalente de arena

AENOR UNE 83. I30-90. Ensayo de azul de metileno.

AENOR UNE 83.124-90. Reactividad de los áridos respecto a los álcalis.
AENOR UNE 83.I I 5. Friabilidad de la arena Norma.

AENOR UNE 83. I 16. Desgaste de la grava, ensayo de los Ángeles. AENOR UNE 83.133. Determinación del peso específico y absorción de agua en arenas. Método del picnómetro.

AENOR UNE 83. I 34. Determinación del peso específico y absorción de agua en gravas. Método de la balanza

AENOR UNE 7.I 36. Estabilidad de áridos frente a disoluciones de sulfato sódico o magnésico.

Arredondo F. "Dosificación de hormigones, manuales y normas". IRTCC. Madrid, 1977

Arredondo F y Soria F. Estudio de materiales. Tomo I. Ed. S.P. Mopu. Revista de Obras Públicas, Madrid 1993.

Comisión Permanente. Instrucción para Proyecto y la ejecución de obras de hormigón en masa o armado (EH-73). Decreto 3062/1973.

Comisión Permanente. Instrucción para Proyecto y la ejecución de obras de hormigón en masa o armado (EH-80).

Comisión permanente del hormigón. Instrucción para el proyecto y la ejecución de obras de hormigón en masa o armado (EH-9|). Real Decreto 824/1988

Chinchón , J.S y Soriano Carrillo, J. "Revisión de la normativa que regula la caracterización de la cantidad de compuestos de azufre en los áridos utilizados en la fabricación de hormigones". Cemento y hormigón n 673 (1989). pp. I I53-1 159. 
Delibes, A. 1989. "Tecnología y propiedades mecánicas del hormigón”. Ed. Intermac, 1989.

Dreux G. "Guía práctica del hormigón". E.T.A de Barcelona, 1981.

Figg J.W y Bowden S.R, 1970. "Análisis de hormigones". Building Research Stration, Departament of the Environment, 1970.

García de Cura, M.A. 1996. "Áridos". Degradación y Conservación del Patrimonio Arquitectónico. Ed. Complutense, S.A, Madrid, 1996.

MELC 4.02-a, del Laboratorio Central de Ensayo de Materiales de Construcción de Madrid.

MELC 4.02-a, del laboratorio Central de Ensayo de Materiales de Construcción de Madrid.

Mopu. Pliego General de Carreteras (PG-3). Ed. Mopu, Madrid, (1975)

Mopu. Diseño de mezclas bituminosas. Ed. Mopu. Madrid, 1986.

Neville A.H. "Tecnología del concreto". Tomo I. Instituto Mexicano del Cemento y del concreto A.C. ed. Limusa, Mexico, D.F. 1984

Norma NE I.32-a.
Norma ASTM. C 142-39.

Norma ASTM C 235-47T.

Norma E 132-b . Instituto Técnico de la Construcción y el Cemento. Norma ASTM C-123-64.

Panet y Toureq (37)Les essais de granulats. Connaissances actueles et orientation des recherches. Revue Général des routes, 465. 1971. pp. 97-108.

Rodríguez Montero, J. 1991. "Comentarios a las prescripciones de los áridos de la Instruccción EH-9|". Ed. S.P. E.U. A.T de Granada, Granada, 1991.

Valverde Espinosa, I. 1993." Caracterización de áridos para hormigones en la Depresión de Granada" Tesis Doctoral. Publicación inédita Universidad de Granada, 1993.

Viruela Bloda A. 1967. "Los áridos en la construcción. Empleo de los áridos calcáreos en morteros y hormigones". Ed. Editores Técnicos Asociados S.A. Barcelona, 1967. pp. 297-336.

Walkey y Black. "Determinación aproximada de la materia orgánica por el método de dicromato potásico. 